\title{
Late Onset of Chronic Granulomatous Disease Revealed by Paecilomyces lilacinus Cutaneous Infection
}

\author{
Clément Lemaigre ${ }^{1,2} \cdot$ Felipe Suarez ${ }^{3} \cdot$ Jean-Philippe Martellosio ${ }^{1,2} \cdot$ Cindy Barbarin ${ }^{2,4} \cdot$ Kévin Brunet $^{2,5,6}$. \\ Jean Claude Chomel ${ }^{7,8}$. Ewa Hainaut ${ }^{4}$. Blandine Rammaert ${ }^{1,2,5}$. France Roblot ${ }^{1,2,5}$. José Miguel Torregrosa-Diaz ${ }^{7}$
}

Received: 10 May 2021 / Accepted: 3 August 2021 / Published online: 1 October 2021

(c) The Author(s) 2021

\begin{abstract}
Chronic granulomatous disease (CGD) is an inherited immunodeficiency due to defective leukocyte NADPH responsible for recurrent infections and aberrant inflammation. Mutations in the $C Y B B$ gene are responsible for the X-linked CGD and account for approximately $70 \%$ of the cases. CGD is diagnosed during childhood in males. Female carriers may have biased $\mathrm{X}$-inactivation and may present with clinical manifestations depending on the level of residual NADPH oxidase activity. We report the case of a previously asymptomatic female carrier who was diagnosed at age 67 with a skin infection with the rare fungus Paecilomyces lilacinus as the first manifestation of CGD. Dihydrorhodamine 123 (DHR) activity was below $10 \%$. Next-generation sequencing (NGS) revealed mutations in DNMT3A, ASXL1, and STAG2 suggesting that clonal hematopoiesis could be responsible for a progressive loss of NADPH oxidase activity and the late onset of X-linked CGD in this patient. Long-term follow-up of asymptomatic carrier women seems to be essential after 50 years old.
\end{abstract}

Keywords Chronic granulomatous disease $\cdot$ clonal hematopoiesis $\cdot$ invasive fungal disease $\cdot$ antifungal prophylaxis

\section{Introduction}

Chronic granulomatous disease (CGD) is a primary immune deficiency associated with reduced NADPH oxidase activity in phagocytes. NADPH oxidase complex is responsible for the production of free radicals leading to oxidative burst

José Miguel Torregrosa-Diaz

jose-miguel.torregrosa-diaz@chu-poitiers.fr

1 Service de Maladies Infectieuses Et Tropicales, CHU Poitiers, Poitiers, France

2 University of Poitiers, Poitiers, France

3 Necker - Enfants Malades, APHP - Centre Université de Paris, Institut Imagine, INSERM UMR 1163 \& CNRS ERL 8254, Université de Paris, Paris, France

4 Service de Dermatologie, CHU Poitiers, Poitiers, France

5 INSERM U1070, Poitiers, France

6 Département Des Agents Infectieux, CHU Poitiers, Poitiers, France

7 Service d'Oncologie Hématologique Et Thérapie Cellulaire, INSERM CIC 1402, CHU Poitiers, 2 rue de la Milétrie, 86021 Poitiers Cedex, France

8 Service de Cancérologie Biologique, Poitiers, France and microorganism killing. The different forms of CGD are associated with inactivating mutations in genes coding for the subunits of the phagocyte NADPH oxidase complex. Subunits Gp91, encoded by $C Y B B$ gene (X-linked), and $\mathrm{p} 22$ encoded by the $C Y B A$ gene (autosomal recessive) are located on the lysosomal membrane. The other subunits encoded by the NCF 1-4 genes (subunits p22, p47, p67, and p40phox) are localized on the cytosolic side of the lysosomal membrane and regulate the catalytic activity of complex [1-3].

The X-linked form is the most common form, usually diagnosed around 5 years old, rarely after 20 [4]. The prognosis is poor due to severe recurrent infections and granulomatous inflammation affecting various organs. Despite antibacterial and antifungal prophylaxis, the mean survival time is 37.8 years in the X-linked form, and 49.6 years in the autosomal recessive form [4]. Although X-linked form preferentially affects men, $8 \%$ of cases occur in women due to unfavorable lyonization or new mutation of the active $\mathrm{X}$ chromosome $[4,5]$.

Today, CGD is diagnosed mostly by quantifying the production of hydrogen peroxide $\left(\mathrm{H}_{2} \mathrm{O}_{2}\right)$ by dihydrorhodamine oxidation (DHR). Susceptibility to infections is apparent in female X-inked CGD carriers with DHR below 20\% (and especially below $10 \%$ ) in particular with opportunistic 
pathogens such as Nocardia spp. and Burkholderia spp., and invasive fungi such as Aspergillus spp. [1-3]. Autoimmune or inflammatory manifestations of the disease such as discoid lupus erythematosus, Raynaud's phenomenon, and granulomatous colitis may be present regardless of the DHR level [5].

We report the case of a female carrier who developed a symptomatic form of X-linked CGD with DHR $<10 \%$ at the age of 67 years old and hypothesized that the development of clonal hematopoiesis $(\mathrm{CH})$ could be responsible for the late-onset disease.

\section{Case Description}

The patient was a 67-year-old woman, known to be an asymptomatic carrier of X-linked CGD (Gp91 subunit mutation, exon 7, c.676C > T, p. Arg226*) following the death of a daughter due to salmonellosis in 1985. She had two other sons with X-linked CGD, one who died in 1988 at the age of 7 from invasive aspergillosis, and one with a history of recurrent bacterial and fungal infections (aspergillosis) and inflammatory bowel disease, alive and well at age of 35 . The patient had never manifested with infections or inflammatory symptoms related to CGD until she was referred to our unit in March 2019. She also had a history of breast cancer treated by tumorectomy, hormonal therapy, and radiotherapy at age 62 years in 2013.

The patient presented with skin lesions of her right thumb in December 2018. She had worked as a sheep farmer for many years. The papules evolved towards pustules with purulent discharge on an inflammatory background since December 2018 despite two different lines of oral antibiotic therapy and valaciclovir. Biopsies were performed in March 2019 after she was referred to our department (Fig. 1). All biopsy cultures grew a mold, Paecilomyces lilacinus, which was confirmed by sequencing. Histopathological examination showed an inflammatory reaction and mycelial elements compatible with invasive fungal disease. Other microbiological investigations remained negative, including poxvirus PCR. The patient was treated with oral voriconazole, loading dose $400 \mathrm{mg}$ BID then $200 \mathrm{mg}$ BID for 3 months. Treatment was well tolerated and allowed complete healing of the lesions. Her family history and the infection with a rare fungus led us to explore the possibility of immune deficiency linked to CGD. DHR was found to be $<10 \%$ consistent with a skewed X-inactivation. No abnormalities in other compartments were detected: Immunophenotypic study of T and NK lymphoid populations revealed a normal quantitative distribution. The B lymphocyte study revealed a discrete B-lymphopenia (5\% (84 cells $/ \mu \mathrm{L}$ ) of CD19+ lymphocytes for a normality interval between 7.2 and $11.2 \%$ ). There was a normal quantitative distribution of $\operatorname{IgA}$, IgM, and IgG. The complement factors were normal. As the patient had no infectious or inflammatory manifestations of X-linked CGD before age 67, no DHR determination was performed before, but we hypothesized that the severely decreased DHR found was the consequence of an acquired skewing of $X$ chromosome. A routine-implemented nextgeneration sequencing (NGS) targeted panel was performed in nucleated bone marrow cells. Included genes and exons are available in Supplemental Table 1. The following variants were found: p.Gln573_Ala575del in DNMT3A with a variant allele frequency (VAF) of 7.88\%, p.Val841 Ala in ASXL1 (VAF 47.94\%), and p.Asp651Gly in STAG2 (VAF $48.26 \%$ ). The patient had normal blood counts and no signs of myelodysplasia were noted on the bone marrow aspirate, consistent with the presence of clonal hematopoiesis. Prolonged secondary prophylaxis with oral posaconazole and cotrimoxazole was indicated to prevent bacterial and fungal infections in this patient with late-onset severe CGD.

\section{Discussion}

This case addresses the issue of a late-onset CGD manifested by an invasive fungal infection due to a rare environmental mold, P. lilacinus, at age 67 in a previously asymptomatic female carrier of X-linked CGD.

Three other cases of late-onset CGD have been described in the literature, all three patients being women aged 45, 53, and 64 [6-8]. These cases were diagnosed
Fig. 1 a Right thumb before treatment. b Right thumb after treatment
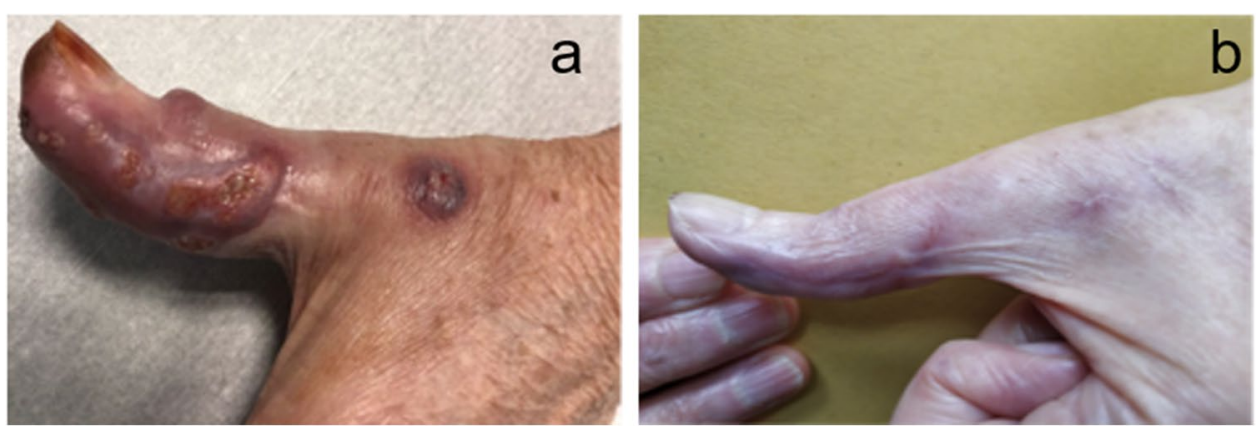
after infection by opportunistic pathogens (Aspergillus spp. [6], pulmonary infection with Burkholderia cepacia [7], and Serratia marcescens [8]). Invasive fungal diseases due to molds other than Aspergillus spp. are rare during CGD course [9]. P. lilacinus is a cosmopolitan saprophytic soil and plant filamentous fungus, with a particular tropism for eyes, skin, and sinuses. The skin is the second most common site of infection after ophthalmic involvement with various unspecific manifestations of the disease from erythematous papules to necrotic lesions or cellulitis [10].

Age-related $\mathrm{CH}-\mathrm{a}$ frequent phenomenon correlated with medullary aging [11-13]-affects roughly $2-3 \%$ of adults over the age of 50 [14]. It is associated with an increased risk of subsequent hematological malignancies and atherosclerosis and could be the key factor to understand the late onset of CGD in our patient. It consists of the emergence of a related cell clone with an acquired gene mutation. The selection of one or more stem cells having a proliferation advantage can gradually replace the other stem cells in some cases [12], leading to reduced genetic diversity of hematopoiesis via the selection of one or more stem cells to the detriment of others. Two major consequences are known to date: increased cardiovascular risk and a variable rate of myelodysplasia and acute leukemia $[15,16]$.

The impact of $\mathrm{CH}$ is however incomplete, as a sort of "incomplete penetrance," so the influence of some extrinsic factors, as an inflammatory environment, has been proposed. There is an enhanced inflammation seen in patients with CGD, with a mechanism not fully understood but likely related to a defective production of ROS leading to increased expression of NFkB-regulated inflammatory genes [17] and high levels of inflammatory mediators that are expressed in monocytes from patients with X-linked CGD without acute infection compared with controls. So, a variety of inflammatory conditions are seen in CGD patients.

The association of $\mathrm{CH}$ and inflammatory/autoimmune disorders has already been reported [18] and there is a growing evidence supporting the association between the XCI skewing and the emergence of autoimmune/non-infectious diseases [19].

Wolach et al. have described for the first time a late onset of X-linked CGD in a 64-year-old woman and have suspected this phenomenon without being able to prove it [8]. In our patient, two of the three mutations most frequently involved in CH (DNMT3A and ASXL1) [20] were found. DNMT3A gene is located on chromosome 2 and encodes DNA (cytosine-5)-methyltransferase $3 \mathrm{~A}$, an epigenetic modifier essential in hematopoietic stem cell differentiation [21]. It may confer a proliferation advantage becoming more important within stem cell aging [22]. ASXL1 gene is an epigenetic marker frequently involved in all subtypes of myeloid malignancies and also in clonal hematopoiesis [23].
$\mathrm{X}$-inactivation is an embryological phenomenon that is permanent at each cell division but whose allelic ratio varies throughout life [24]. The reasons of this different $\mathrm{XCI}$ with time remain unclear. In this setting, the silencing by long non-coding RNA Xist (which variation with time is unknown) and the hematopoietic stem cell senescence remain a valid hypothesis to clarify [25], but their roles have to be determined. As another potential mechanism, the allele-specific loss of methylation with time has been also proposed [25] and could fit best with our hypothesis of the role of clonal hematopoiesis.

Due to the absence of any significant infection until the age of 67 and the depth of the deficit detected in our patient, we hypothesize that it is unlikely that she has had a so deep impaired DHR since birth. The skewed X-inactivation in favor of the mutated $C Y B B$ gene could have led to the progressive appearance of the genotype and phenotype disease. This asymmetry could be explained by the age-related selection of a cell carrying the mutation [23], as in our patient we found probable $\mathrm{CH}$, so a plausible explanation could be that if the predominant clone carries the $C Y B B$ mutation, the pattern of inactivation of $\mathrm{X}$ becomes asymmetric in favor of this mutation. That would have led to the progressive reduction of the DHR and thus the acquisition of the disease. Interestingly, the patient had been treated by radiotherapy 5 years before, which might have accelerated the acquisition of $\mathrm{CH}$.

Our finding suggests that asymptomatic carriers of $C Y B B$ mutations should have life-long clinical and biological surveillance. A yearly clinical exam and DHR measurement should start at 50 years old when the risk of clonal hematopoiesis becomes significant, or before if the patient starts to have inflammatory symptoms. Inflammatory symptoms like lupus discoid, pulmonary fibrosis, and Crohn-like disease, can appear before infection when DHR is $>20 \%$. Antifungal and antibacterial prophylaxis should be started as soon as the DHR is $<20 \%$, a percentage below which the risk of infection prevails [5].

In conclusion, the X-linked form of CGD could affect older women due to clonal hematopoiesis. Close monitoring of women carrying the mutation by studying phagocytic function should be interesting to detect DHR decrease below $20 \%$. This strategy could help to prevent infections by starting early chemoprophylaxis and environmental prevention measures.

Supplementary Information The online version contains supplementary material available at https://doi.org/10.1007/s10875-021-01140-1.

Author Contribution CL and JT wrote the manuscript. JCC performed NGS analysis. CL, FR, JT, EH, and BR were in charge in the patient and collected the clinical data. FS has apported the original idea and the clinical and biological information of the patient's sons. KB and $\mathrm{CB}$ have performed molecular analysis leading to the identification of 
the fungus. All authors have contributed to the redaction of this work and have approved the final version.

Data availability Every data used of this work is available upon request.

Code Availability Not applicable.

\section{Declarations}

\section{Ethics Approval Not applicable.}

Consent to Participate The patient consents to the use of her clinical information to the redaction of this case report.

Consent for Publication The patient formally consented to the use of her clinical information to be published for this case report

Conflict of Interest The authors declare no competing interests.

Open Access This article is licensed under a Creative Commons Attribution 4.0 International License, which permits use, sharing, adaptation, distribution and reproduction in any medium or format, as long as you give appropriate credit to the original author(s) and the source, provide a link to the Creative Commons licence, and indicate if changes were made. The images or other third party material in this article are included in the article's Creative Commons licence, unless indicated otherwise in a credit line to the material. If material is not included in the article's Creative Commons licence and your intended use is not permitted by statutory regulation or exceeds the permitted use, you will need to obtain permission directly from the copyright holder. To view a copy of this licence, visit http://creativecommons.org/licenses/by/4.0/.

\section{References}

1. Kuhns DB, Alvord WG, Heller T, Feld JJ, Pike KM, Marciano $\mathrm{BE}$, et al. Residual NADPH oxidase and survival in chronic granulomatous disease. N Engl J Med. 2010;363:2600-10.

2. Winkelstein JA, Marino MC, Johnston RB, Boyle J, Curnutte J, Gallin JI, et al. Chronic granulomatous disease: report on a national registry of 368 patients. Medicine. 2000;79:155-69.

3. Holland SM. Chronic granulomatous disease. Hematol Oncol Clin North Am. 2013;27:89-99.

4. van den Berg JM, van Koppen E, Åhlin A, Belohradsky BH, Bernatowska E, Corbeel L, et al. Chronic granulomatous disease: the European experience Alspaugh A, editor. PLoS ONE. 2009;4:e5234.

5. Marciano BE, Zerbe CS, Falcone EL, Ding L, DeRavin SS, Daub J, et al. X-linked carriers of chronic granulomatous disease: illness, lyonization, and stability. J Allergy Clin Immunol. 2018;141:365-71.

6. Rösen-Wolff A, Soldan W, Heyne K, Bickhardt J, Gahr M, Roesler J. Increased susceptibility of a carrier of X-linked chronic granulomatous disease (CGD) to Aspergillus fumigatus infection associated with age-related skewing of lyonization. Ann Hematol. 2001;80:113-5.

7. Sarwar G, Malmanche T, Rassam L, Grainge C, Williams A, Arnold D. Chronic granulomatous disease presenting as refractory pneumonia in late adulthood. Respirol Case Rep. 2015;3:54-6.

8. Wolach B, Scharf Y, Gavrieli R, de Boer M, Roos D. Unusual late presentation of $\mathrm{X}$-linked chronic granulomatous disease in an adult female with a somatic mosaic for a novel mutation in CYBB. Blood. 2005;105:61-6.
9. Henriet S, Verweij PE, Holland SM, Warris A. Invasive fungal infections in patients with chronic granulomatous disease. In: Curtis N, Finn A, Pollard AJ, editors. Hot topics in infection and immunity in children IX [Internet]. New York, NY: Springer New York; 2013 [cited 2021 May 6]. p. 27-55. Available from: http:// link.springer.com/https://doi.org/10.1007/978-1-4614-4726-9_3

10. Pastor FJ, Guarro J. Clinical manifestations, treatment and outcome of Paecilomyces lilacinus infections. Clin Microbiol Infect. 2006;12:948-60.

11. Shlush LI. Age-related clonal hematopoiesis. Blood. 2018;131:496-504.

12. Groarke EM, Young NS. Aging and hematopoiesis. Clin Geriatr Med. 2019;35:285-93.

13. Zink F, Stacey SN, Norddahl GL, Frigge ML, Magnusson OT, Jonsdottir I, et al. Clonal hematopoiesis, with and without candidate driver mutations, is common in the elderly. Blood. 2017; 130:742-52.

14. Steensma DP. Clinical consequences of clonal hematopoiesis of indeterminate potential. Blood Adv. 2018;2:3404-10.

15. Sano S, Wang Y, Walsh K. Clonal hematopoiesis and its impact on cardiovascular disease. Circ J. 2018;83:2-11.

16. Desai P, Hassane D, Roboz GJ. Clonal hematopoiesis and risk of acute myeloid leukemia. Best Pract Res Clin Haematol. 2019;32:177-85.

17. Brown KL, Bylund J, MacDonald KL, Song-Zhao GX, Elliott MR, Falsafi R, et al. ROS-deficient monocytes have aberrant gene expression that correlates with inflammatory disorders of chronic granulomatous disease. Clin Immunol. 2008;129:90-102.

18. Arends CM, Weiss M, Christen F, Eulenberg-Gustavus C, Rousselle A, Kettritz R, et al. Clonal hematopoiesis in patients with anti-neutrophil cytoplasmic antibody-associated vasculitis. Haematologica. 2020;105:e264-7.

19. Simmonds MJ, Kavvoura FK, Brand OJ, Newby PR, Jackson LE, Hargreaves CE, et al. Skewed X chromosome inactivation and female preponderance in autoimmune thyroid disease: an association study and meta-analysis. J Clin Endocrinol Metab. 2014;99:E127-31.

20. Jaiswal S, Fontanillas P, Flannick J, Manning A, Grauman PV, Mar BG, et al. Age-related clonal hematopoiesis associated with adverse outcomes. N Engl J Med. 2014;371:2488-98.

21. Challen GA, Sun D, Jeong M, Luo M, Jelinek J, Berg JS, et al. Dnmt3a is essential for hematopoietic stem cell differentiation. Nat Genet. 2012;44:23-31.

22. Buscarlet M, Provost S, Zada YF, Barhdadi A, Bourgoin V, Lépine $\mathrm{G}$, et al. DNMT3A and TET2 dominate clonal hematopoiesis and demonstrate benign phenotypes and different genetic predispositions. Blood. 2017;130:753-62.

23. Busque L, Mio R, Mattioli J, Brais E, Blais N, Lalonde Y, et al. Nonrandom $\mathrm{X}$-inactivation patterns in normal females: lyonization ratios vary with age. Blood. 1996;88:59-65.

24. Mengel-From J, Lindahl-Jacobsen R, Nygaard M, Soerensen M, Ørstavik KH, Hertz JM, et al. Skewness of X-chromosome inactivation increases with age and varies across birth cohorts in elderly Danish women. Sci Rep. 2021;11:4326.

25. Hatakeyama $\mathrm{C}$, Anderson $\mathrm{C}$, Beever $\mathrm{C}$, Peñaherrera $\mathrm{M}$, Brown $\mathrm{C}$, Robinson W. The dynamics of $\mathrm{X}$-inactivation skewing as women age: XCI skewing and age. Clin Genet. 2004;66:327-32.

Publisher's Note Springer Nature remains neutral with regard to jurisdictional claims in published maps and institutional affiliations. 